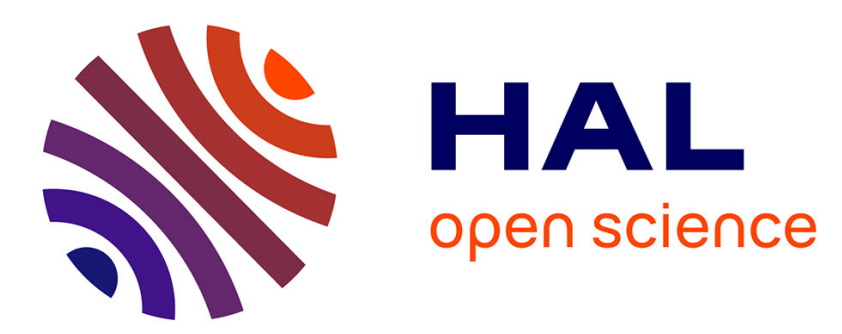

\title{
Ligand-protected gold nanoclusters probed by IRMPD spectroscopy and quantum chemistry calculations
}

Nicolas Nieuwjaer, Ali Beydoun, Frédéric Lecomte, Bruno Manil, Debora

Scuderi, Charles Desfrançois

\section{- To cite this version:}

Nicolas Nieuwjaer, Ali Beydoun, Frédéric Lecomte, Bruno Manil, Debora Scuderi, et al.. Ligandprotected gold nanoclusters probed by IRMPD spectroscopy and quantum chemistry calculations. 2021. hal-02908285v2

\section{HAL Id: hal-02908285 \\ https://hal.science/hal-02908285v2}

Preprint submitted on 19 Oct 2021

HAL is a multi-disciplinary open access archive for the deposit and dissemination of scientific research documents, whether they are published or not. The documents may come from teaching and research institutions in France or abroad, or from public or private research centers.
L'archive ouverte pluridisciplinaire HAL, est destinée au dépôt et à la diffusion de documents scientifiques de niveau recherche, publiés ou non, émanant des établissements d'enseignement et de recherche français ou étrangers, des laboratoires publics ou privés. 


\title{
Ligand-protected gold nanoclusters probed by IRMPD spectroscopy and quantum chemistry calculations
}

N. Nieuwjaer ${ }^{1}$, A. Beydoun 1 , F. Lecomte ${ }^{1}$, B. Manil1 ${ }^{1}$, D. Scuderi ${ }^{2}$ and C. Desfrançois ${ }^{1 a)}$

1 Université Sorbonne Paris Nord, Lab. de Physique des Lasers, CNRS, 93430 Villetaneuse, France

2 Université Paris Saclay, Institut de Chimie Physique, UMR 8000 CNRS, 91898 Orsay, France

a) Author to whom correspondence should be addressed: charles.desfrancois@univ-paris13.fr

\begin{abstract}
This paper reports an attempt to structurally characterize isolated ligand-protected gold nanoclusters by means of gas-phase InfraRed Multiple Photon Dissociation (IRMPD) spectroscopy compared to quantum chemistry Density Functional Theory (DFT) calculations. The mass-selected kilodalton nanocluster complexes consist of ten or eleven gold atoms that are bound to glutathione or phosphine ligands and are produced by ElectroSpray lonization (ESI) in the form of multiply charged anions or cations. This study allows us to build some methodology benchmarks for species that are large for IRMPD experiments and that are used for biochemistry applications. These gas-phase results on isolated ions are compared to condensed phase data from Fourier-Transform InfraRed (FTIR) spectroscopy and to theoretical IR spectra that are calculated with two different functional/basis sets, namely B3LYP/6-31G* and M06L/LanL2DZ, at the scaled static harmonic level. Although theoretical calculations are able to reproduce well the experimental IR spectra, the size of such species and the presence of many possible interactions between ligands make difficult a precise assignment among the many possible molecular arrangements.
\end{abstract}

Keywords: ligand-protected gold nanoclusters; IRMPD spectroscopy; DFT calculations. 


\section{INTRODUCTION}

Ultra-small nanoparticles (USNP) and especially metal-ligand nanoclusters (NC) have recently attracted more and more attention, due to their optical [Lin, Shang, Cortie] and magnetic [Jung] properties coupled to their potential for targeting and vectorization of bioactive molecules within living cells [Cobley] and their potential applications in biochemistry and biomedicine [Jiang, Jin]. In the condensed phase, their structural characterization is often performed with X-ray crystallography or NMR and IR methods, but data interpretations are sometimes difficult because of the relative lack of control of their stoichiometry and of their ligand coverage [Zhang]. This is especially true for USNP (<1 nm in diameter) whose chemical/physical properties are very dependent on their size and structure and are intermediate between those of the isolated metal and ligand and those of the liganded nanoparticles in solution [Lu, Shang].

Following a bottom-up approach, we recently published a study of isolated metal-ligand clusters, containing few metal atoms ( $\mathrm{Al}, \mathrm{Fe}, \mathrm{Ru})$ and few anionic acetylacetonate ligands [Nieuwjaer], which allowed for a benchmarking of InfraRed Multiple Photon Dissociation (IRMPD) spectroscopy combined with Density Functional Theory (DFT) quantum chemistry calculations useful for their structural characterization in the gas phase. In the present study, we want to explore to which extent the same methodology can be applied to larger metal-ligand NC with the same success.

Within the last 30 years, IRMPD spectroscopy has been recognized as a powerful tool for structural characterization of very diverse isolated and mass-selected molecular ions, including metal-ligand ionic complexes [Jasikova, Woodin, Maitre], through the comparison of infrared signatures with model spectra that are predicted by DFT calculations [Katari]. Up to now, these IR spectroscopy studies have been however restricted to complexes containing only one or two metal ions and few ligands. Previous experimental data on large kilodalton mass-selected and isolated species have nevertheless been successfully reported on model biomolecules such as small proteins or DNA strands [Gonzalez, Gabelica] in the IR domain, or on ligand-protected metal NC [Veenstra, Hirata, Cirri, Greisch, Bertorelle, Koyasu] in the electronic domain and mainly through photoelectron spectroscopy of anions or photodissociation spectroscopy of cations.

In the present studies, we investigated two different NC that we were able to obtain in the form of the most possible monodisperse solution that can be used in an ElectroSpray Ionisation (ESI) source. The first one is a commercial so-called $0.8 \mathrm{~nm}$ undecagold (Nanoprobes Inc.), which is based 
on a small gold core with covalently bound triphenylphosphine (TPP) ligands. The second sample was synthesized by the Dugourd's group (ILM, Lyon, France) who has already published UV-Vis spectroscopy experiments and calculations on these complexes [Bertorelle, Antoine]: it consists of chained covalent gold-glutathione complexes containing mainly ten gold atoms. Both complexes have a molecular weight of the order of $5 \mathrm{kDa}$, they can be dissolved in water or methanol and, after ESI and transfer into high vacuum, they both produce high intensities of moderately multiply charged ions so that they can be mass-selected at moderate $\mathrm{m} / \mathrm{z}$ ratio $(1-2 \mathrm{k})$.

To our knowledge, the present study constitutes the first one dealing with such isolated massselected large metal-ligand nanoclusters using combined IRMPD/DFT techniques for testing their ability to provide structural information.

\section{EXPERIMENTAL AND THEORETICAL METHODS}

Gold-TPP complexes were purchased from a commercial supplier (Nanoprobes Inc., Yaphank, NY, USA) in the form of a $50 \mathrm{nmol}$ lyophilized non-functionalized $0.8 \mathrm{~nm}$ undecagold and used without further purification in a pure methanol solution at a concentration of 5-10 $\mu \mathrm{M}$. For complementary experiments on gold-TPP complexes, we also bought $\mathrm{Au}(\mathrm{TPP}) \mathrm{Cl}$ (chloro(triphenylphosphine)gold(I) from Sigma-Aldrich) that we used without further purification in a methanol-acetonitrile solution $(80 / 20)$ at a $10^{-4} \mathrm{M}$ concentration. Gold-glutathione complexes were kindly provided by F. Bertorelle in the form of several $\mathrm{mg}$ of lyophilized synthesized samples [Bertorelle] that were used without purification in 1:1 water/methanol solutions at concentrations of about $10 \mu \mathrm{M}$.

IRMPD experiments have been performed at the Free Electron Laser (FEL) of the Centre Laser Infrarouge d'Orsay (CLIO) facility, in the $400-1900 \mathrm{~cm}^{-1}$ spectral range. Ion spectra were recorded using the available $7 \mathrm{~T}$ hybrid FT-ICR mass spectrometer (APEX-Qe, Bruker) coupled to the CLIO beamline. This experimental setup has been previously described in detail [Prazeres, Bakker]. Briefly, ions in solution were delivered in the gas-phase by an ESI source with a typical extraction voltage of $4 \mathrm{kV}$ and a desolvation temperature of $150-200{ }^{\circ} \mathrm{C}$. Ions were accumulated, pre-massselected and thermalized in a quadrupole-hexapole interface and then pulse extracted towards the FT-ICR cell maintained under high vacuum $\left(<10^{-9} \mathrm{mbar}\right)$ and at room temperature. Mass-selected ions were stored and irradiated for 1 to 4 seconds with the IR-FEL light in the frequency range of 
interest. Depending on the electron energy used (35-45 MeV) for covering a given spectral range, the average CLIO laser power is recorded and lies in between 0.5 and $1.2 \mathrm{~W}$. Its frequency resolution (about $1 \%$ or better) and the spectral rotational broadening of ions at $300 \mathrm{~K}$ lead to an overall linewidth of at least $10 \mathrm{~cm}^{-1}$. In order to increase fragmentation for these large and strongly bound systems, ions were often also submitted to an auxiliary broad-band $\mathrm{CO}_{2}$ laser (Universal Laser System, $10 \mathrm{~W}$, cw operation centered at $\lambda=10.6 \mu \mathrm{m})$ synchronized with the CLIO laser, at the beginning of the irradiation period and for times varying between few and few tens of milliseconds. The $\mathrm{CO}_{2}$ laser pulse length was adjusted for each experiment to avoid photo-dissociation of the molecule by the $\mathrm{CO}_{2}$ laser alone while promoting the fragmentation in the presence of the CLIO laser.

When the IR-FEL light is in resonance with an active vibrational mode of the mass-selected ions, multiple and sequential events of photon absorption and intramolecular vibrational energy redistribution (IVR) ultimately activate their fragmentation. At each spectral wavenumber, typically five mass spectra were recorded and accumulated in order to compare the integrated number of precursor $\left(I_{p}\right)$ and fragment $\left(I_{f}\right)$ ions as a function of the laser frequency. An IRMPD spectrum is then obtained by recording the photofragmentation yield, $R=-\ln \left[I_{p} /\left(I_{p}+\sum I_{f}\right)\right]$ as a function of the wavenumber of the IR-FEL radiation.

We also performed Fourrier-Transform IR (FTIR) experiments on the two samples, either in transmission in a $\mathrm{KBr}$ matrix or by using the Attenuated Total Reflectance (ATR) technique on a thin film of solid sample evaporated on a ZnSe window, with a Thermo Scientific Nicolet 380 FTIR spectrometer.

All static DFT calculations have been performed using the Gaussian 16 package [Frisch] on the HPC platform MAGI of Université Sorbonne Paris Nord. Full geometry optimizations of the all-atoms NC have been performed starting from trial structures inspired either by that of the crystalline phase of a similar species for gold-TPP NCs [McKenzie] or by previous calculations [Bertorelle] for goldglutathione NC. We used the two functionals/basis sets B3LYP/6-31G* [Becke, Lee, Ditchfield], which has proved to be accurate in our previous studies [Nieuwjaer], and M06L/LanL2DZ [Zhao, Hay], which is supposed to give better energies for metal chemistry [Cramer]. For Au atoms, we used in both cases the LanL2DZ [Hay] basis set which includes an effective core potential for heavy 
transition metals. Since no spin effect is expected with gold atoms, all calculations were performed at the singlet spin state. For understanding where the charges are localized in the studied ions, we also performed natural population analysis (NPA) at equilibrium geometries.

Frequency analysis was performed at the same levels of theory in the harmonic approximation. The size of these species requires too much memory to use the default analytical method for computing the second derivatives of the energy, so that we had to use a numerical method (freq=numer option, as implemented in Gaussian 16), which makes frequency calculations very time-consuming (about 3 months on one node of our platform). For comparison with the measured IRMPD spectra, the calculated vibrational line spectra were scaled by the same factor that we adopted in our previous study on metal-Acac complexes [Nieuwjaer], i.e. 0.975 for both B3LYP and M06L calculations, and they were convoluted with a Lorentzian line shape function with a full width at half-maximum (FWHM) value of $10 \mathrm{~cm}^{-1}$.

\section{RESULTS AND DISCUSSION}

\section{A. MASS SPECTROMETRY}

The formula of the purchased non-functionalized $0.8 \mathrm{~nm}$ undecagold gold-TPP NC is given to be of the form $\mathrm{Au}_{11}\left(\mathrm{PAr}_{3}\right)_{7} \mathrm{Cl}_{3}^{-}$where $\mathrm{Ar}=\mathrm{C}_{6} \mathrm{H}_{4}-\mathrm{CO}-\mathrm{NH}-\mathrm{CH}_{3}$, i.e. a N-methylphenylamide radical. From this formula, it was expected that intact tri-cations $\left[\mathrm{Au}_{11}\left(\mathrm{PAr}_{3}\right)_{7}\right]^{3+}$ could be observed after removal of the three chlorine anions during electrospraying. Tri-cations where indeed observed but the main mass peak corresponds to complexes with one more TPP ligand, i.e. $8\left(\operatorname{PAr}_{3}\right)$ ligands instead of 7. However, the seven-ligands complexes can be obtained as the main mass peak by Collision Induced Dissociation (CID) in the quadrupole-hexapole interface of the FT-ICR spectrometer as displayed in Figure 1 (see also Fig. 1 of the supplementary material for a full mass spectrum). We note that the presence of both 7- and 8-TPP ligands protecting undecagold NC has already been shown and discussed in the context of ligand exchange pathways to form larger gold NC [McKenzie]. The exact composition of ions produced here by ESI is however not straightforward, since the observed masses are higher than those expected from the given formula (5198.721 for $\left.\operatorname{Au}_{11}\left(\operatorname{PAr}_{3}\right)_{7}\right)$. As displayed in figure 1, the isotopic distribution can be well fitted with a formula containing seven more methyl groups (seven $\mathrm{CH}_{3}$ replacing seven $\mathrm{H}$ atoms, i.e. $+98 \mathrm{Da}$ ). Since this number of extra methyl 
groups is equal to the number of ligands, we assume that they are localized on each ligand (one Nethylphenylamide radical replacing one $\mathrm{N}$-methylphenylamide radical over the three of the ligand), i.e. $\mathrm{PAr}_{3} \mathrm{CH}_{2}$ instead of $\mathrm{PAr}_{3}$.

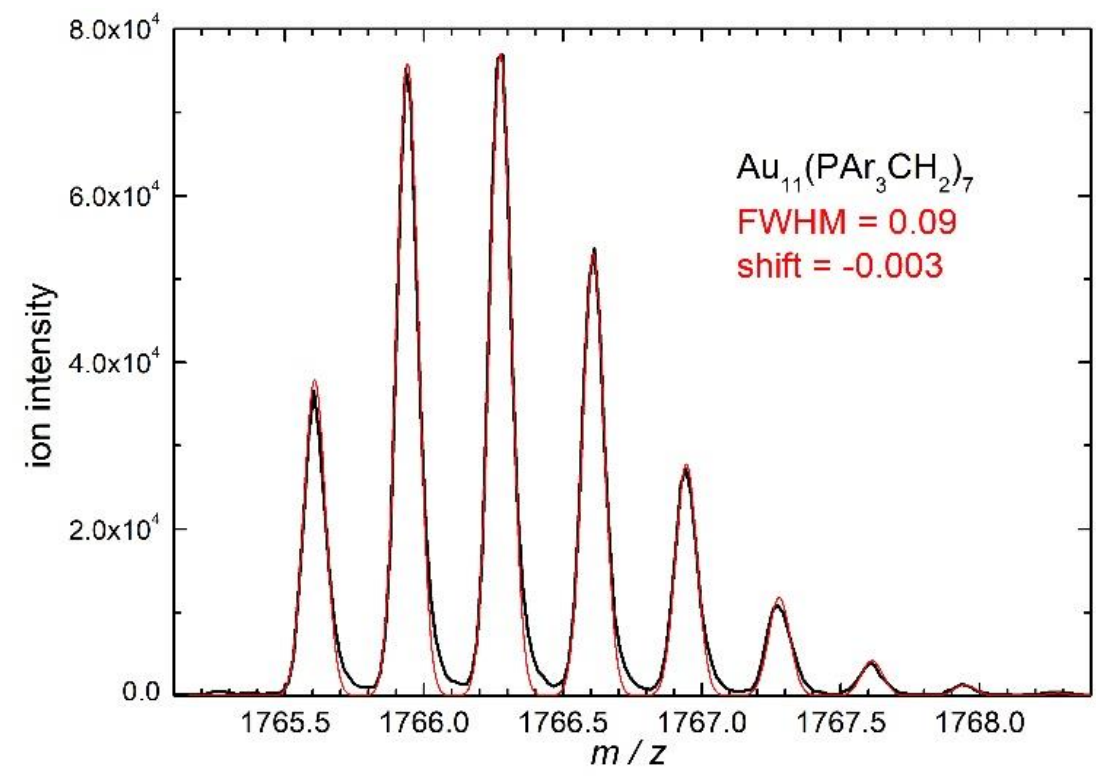

FIG. 1: Experimental (black) and fitted (red) typical mass spectrum; the fitted curve corresponds to the formula with seven extra methyl groups, one on each TPP ligands; the fitting curve has been calculated with the indicated FWHM linewidth and shift that are typical of the FTICR-MS instrument.

From this comparison, it appears that both absolute positions and relative intensities match well with the assigned formula and the fact that one extra methyl group is present on each ligand may be due to the fact that these undecagold NC are also available with a functionalization that is added on one of the phenylamide group via a short alkane linker on the nitrogen atom. In the following, IR spectra calculations are thus performed on the assumed detected species at $\mathrm{m} / \mathrm{z} 1766$ with the formula $\left[\mathrm{Au}_{11}\left(\mathrm{PAr}_{3} \mathrm{CH}_{2}\right)_{7}\right]^{3+}$.

When using the $\mathrm{Au}(\mathrm{TPP}) \mathrm{Cl}$ solution, we observe mainly a mass peak at $m / z 721$, corresponding to $\left[\mathrm{Au}(\mathrm{TPP})_{2}\right]^{+1}$. Under CID, we observe a signal at $\mathrm{m} / \mathrm{z} 477$ together with a much smaller signal at $\mathrm{m} / \mathrm{z}$ 459 that correspond to the hydrated or not mono-ligand $\left[\mathrm{Au}(\mathrm{TPP})_{1}(\mathrm{H} 2 \mathrm{O})_{1} \text { or }\right]^{+1}$ cation, with no doubt on these compositions. When a droplet of acetic acid is added to the solution, only the $\mathrm{m} / \mathrm{z} 263$ cation, which corresponds to protonated TPP, is mainly observed.

The formula of the gold-glutathione NC has been established in previous studies using the same samples [Bertorelle]: they correspond to a molecular chain of Au-SG, containing mainly ten of such motives and appearing in the form of deprotonated anions after ESI. We here obtain the same mass spectra (see Fig. 2 of the supplementary material) and the isotopic profile at $\mathrm{m} / \mathrm{z} 1256$ fits the formula $\left[(\text { AuSG })_{10}-4 \mathrm{H}^{+}\right]^{4-}$ corresponding to the monoisotopic mass of $5026.40 \mathrm{Da}$. 
Note that we also observed some triply deprotonated anions and some 11- and 12-gold-glutathione complexes, which are less intense and have not been studied here, together with smaller ions as $\left[\text { AuSG- } \mathrm{H}^{+}\right]^{1-}$, which has been studied as a reference complex, and $\left[(\mathrm{AuSG})_{4}-2 \mathrm{H}^{+}\right]^{2-}$.

\section{B. IRMPD SPECTROSCOPY AND COMPARISON WITH FTIR AND CALCULATED SPECTRA}

\section{Gold-triphenylphosphine complexes}

Figure 2 displays a comparison between the FTIR spectrum of the solid neutral sample in $\mathrm{KBr}$ pellets and the IRMPD spectrum obtained on undecagold triply charged cations with seven ligands at $\mathrm{m} / \mathrm{z}$ 1766. Experimental data display two or three runs that have been performed in different frequency ranges and at different IR-FEL laser powers and $\mathrm{CO}_{2}$ laser pre-heating durations so that relative band intensities may not reflect exactly the IR absorption yields.

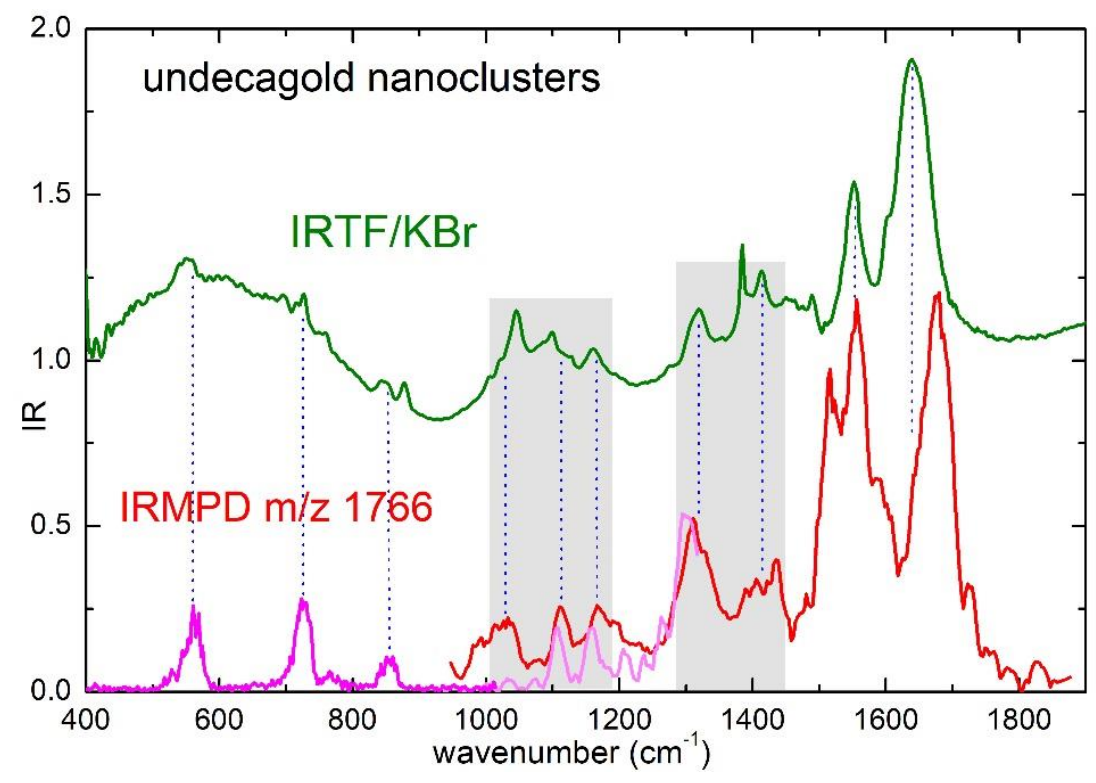

FIG. 2: Experimental IRMPD spectra for the 7-ligands tri-cations (red and magenta) at $\mathrm{m} / \mathrm{z} 1766$, compared to the FTIR spectrum (green) obtained in $\mathrm{KBr}$ pellets on the solid neutral sample $\left[\mathrm{Au}_{11}\left(\mathrm{PAr}_{3}\right)_{7} \mathrm{Cl}_{3}\right]$.

Obviously, these two spectra share several common features but also some differences. The main peak of the FTIR spectrum at $1640 \mathrm{~cm}^{-1}$, which can be attributed to an Amide I band, is also present in the IRMPD spectrum at blue-shifted frequencies. The second peak at $1555 \mathrm{~cm}^{-1}$, which can be attributed to Amide II bands, also appears in IRMPD experiments but as a double-band shape. Following on the red side, a band in between 1300 and $1450 \mathrm{~cm}^{-1}$ also appears in the IRMPD spectrum but with different shapes and a more salient peak at $1290-1310 \mathrm{~cm}^{-1}$. The next wide band, in between 1000 and $1200 \mathrm{~cm}^{-1}$, also appears in IRMPD results but it is weak and in a range where signals are fluctuating. Those two last wide bands should correspond to Amide III bands that are 
known to be very sensitive to the changes in molecular conformations. They thus may appear differently in the solid state and in the isolated cations.

Interestingly, in the low-frequency range, the three peaks observed in the IRMPD spectrum at 560, 720 and $855 \mathrm{~cm}^{-1}$ are also present in the FTIR spectrum at almost the same frequencies but on a large background band whose origin is discussed later. The corresponding vibrational modes probably involve combinations between amide and TPP motions that are more internal and thus less sensitive to the outer interactions of the nanocluster.
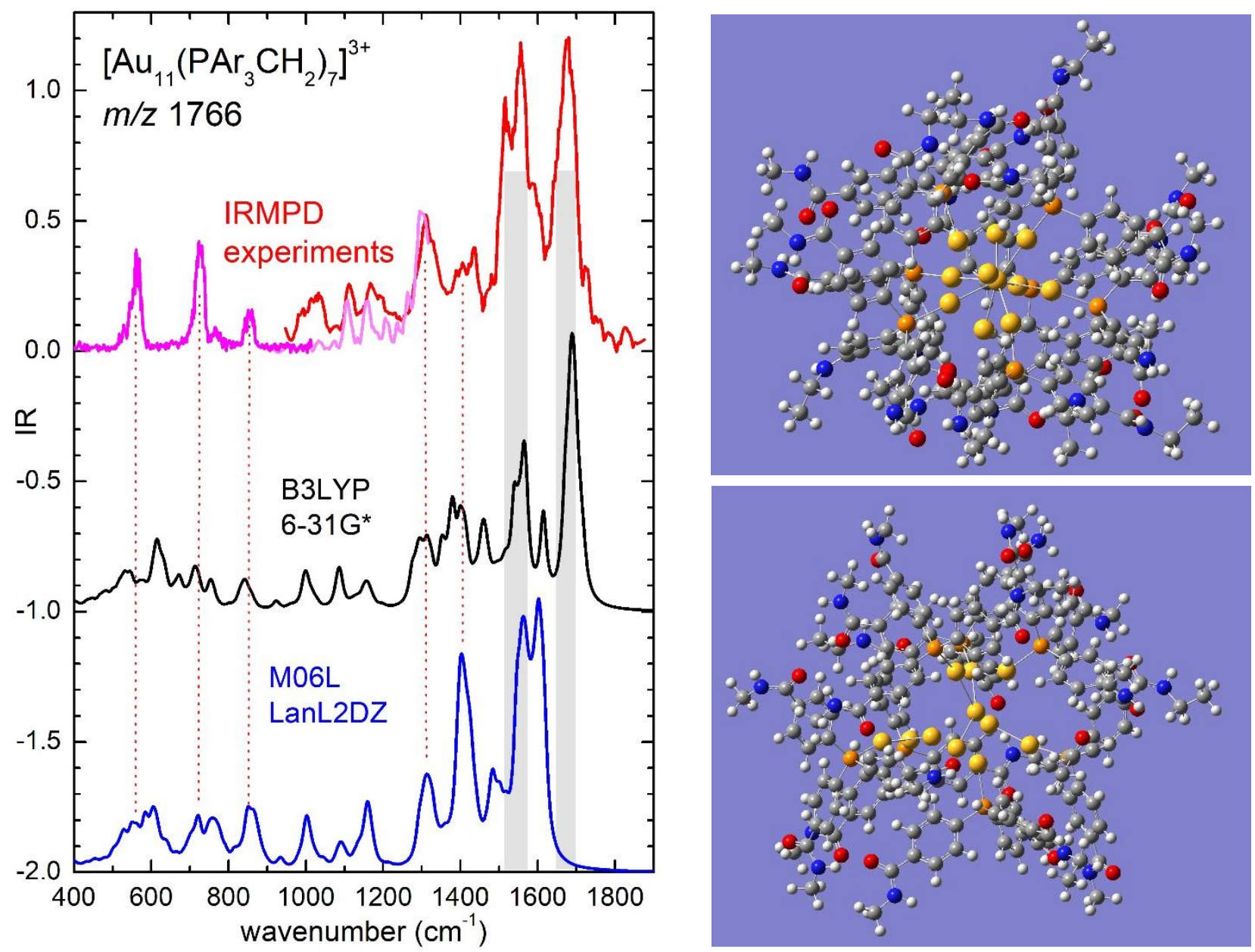

FIG. 3: Comparison between experimental IRMPD spectrum (red and magenta) at $m / z 1766$ (as in figure 2) and calculated spectra for the 7-ligands ions at the M06L/LanL2DZ (blue) and the B3LYP/6-31G* (black) levels. On the right, equilibrium structures are shown as obtained with M06L/LanL2DZ calculations (top), with a preserved gold core, and with B3LYP/6-31G* calculations (bottom), with a less compact gold core (see text for discussion).

Figure 3 displays the comparison between experimental data for 7-ligands undecagold ions at $\mathrm{m} / \mathrm{z}$ 1766 and calculated spectra for $\left[\mathrm{Au}_{11}\left(\mathrm{PAr}_{3} \mathrm{CH}_{2}\right)_{7}\right]^{3+}$ at the M06L/LanL2DZ and B3LYP/6-31G* levels. Calculated IR intensities being quite different from one method to another, they are here scaled so that the main peak intensity is normalized to about 1 in this figure.

Calculated spectra fairly well reproduce the overall experimental spectrum but there are also several discrepancies. In the range of the Amide bands $\left(1200-1650 \mathrm{~cm}^{-1}\right)$, the first two peaks are well 
reproduced by B3LYP calculations while the first one is found much too red-shifted by M06L calculations. As it will be confirmed below, the M06L method seems to fail to reproduce this Amide I vibrational mode. The third salient peak at $1310 \mathrm{~cm}^{-1}$ is also well reproduced in both calculations but these also show up a high peak at $1380-1410 \mathrm{~cm}^{-1}$ that is blue-shifted and wider in the experimental spectrum. In the intermediate range, calculations display three peaks at 1000,1090 and $1150 \mathrm{~cm}^{-1}$ but, as already outlined above, experimental data are not so clear in this frequency range. In the low-frequency part, the three clear experimental peaks are quite well reproduced and M06L seems to perform better than B3LYP in this spectral region. However, the calculated spectra are richer in this range, in particular with an additional peak at $600-620 \mathrm{~cm}^{-1}$, which mainly corresponds to an outof-plane bending of $\mathrm{NH}$ bonds but which does not appear on the right-hand side of the experimental peak at $560 \mathrm{~cm}^{-1}$. Note that all three low-frequency modes at 560, 720 and $855 \mathrm{~cm}^{-1}$ correspond in our calculations also to the out-of-plane bending of $\mathrm{NH}$ but couples to different out-of-plane bending modes of the aromatic hydrogens. We note that the calculated spectra in this region also display a large background which results from the overlap of many different other modes and which is in qualitative agreement with the FTIR spectrum (Fig. 2).

Figure 3 also displays the equilibrium structures for this triply charged cation as they result from M06L or B3LYP calculations, starting from the structure derived from the experimental crystal structure of the same NC but with pure TPP ligands, as previously reported [McKenzie, CCDC1026811]. With M06L, the optimization did not perturb too much the gold core that remains with one central gold atom bound to the ten other peripheral gold atoms. The resulting structure then remains rather compact (top right of Fig. 3), with distances between the central atom and all the other ten gold atoms that lie in the range 2.705 to $2.805 \AA$, i.e. only slightly larger than in the crystal structure where these distances are $2.71 \AA$ for gold atoms also bound to chloride and $2.61 \AA$ for those bound to TPP. On the other hand, upon optimization with B3LYP functional, the gold core starts to fall apart and to form more planar rather than spherical structures (bottom right of Fig. 3), with some Au-Au bonds broken and with a more extended geometry, i.e. a maximal distance between two opposite gold atoms of almost $8 \AA$, and ligands that are also slightly less packed. In both cases, there are many $\mathrm{H}$-bond, electrostatic and Van der Waals interactions between the seven ligands, especially those involving the amide groups, but the Au-P distances are almost the same, 
i.e. $2.45 \pm 0.025 \AA$ ( $2.28 \AA$ in the crystal). Concerning the partial charges on gold atoms, they differ quite a lot between the two calculations and structures: in M06L calculations, the central gold atom holds a negative charge close to $-2(-1.84)$ while the other gold atoms bound to it possess various small positive charges in between +0.06 and +0.23 , whatever they are also bound to a phosphorus ligand or not ; in B3LYP calculations, with no more central gold atom, the partial charges vary in between -0.70 and +0.17 , without a clear qualitative explanation. In both cases, the total charge held by the undecagold NC is slightly negative, i.e. -0.51 for M06L and -0.11 for B3LYP calculations, and all phosphorus atoms hold a charge that is close to $+1(+0.94 \pm 0.03$ for M06L and $+1.03 \pm 0.02$ for B3LYP calculations), so that the total ion charge +3 mainly comes from the seven ligands.

Figure 4 displays complementary experiments and calculations performed on the $\left[\mathrm{Au}(\mathrm{TPP})_{2}\right]^{+1}$ monocation $(\mathrm{m} / \mathrm{z}$ 721) which is made of only one gold atom bound to two non-functionalized triphenylphosphine ligands.

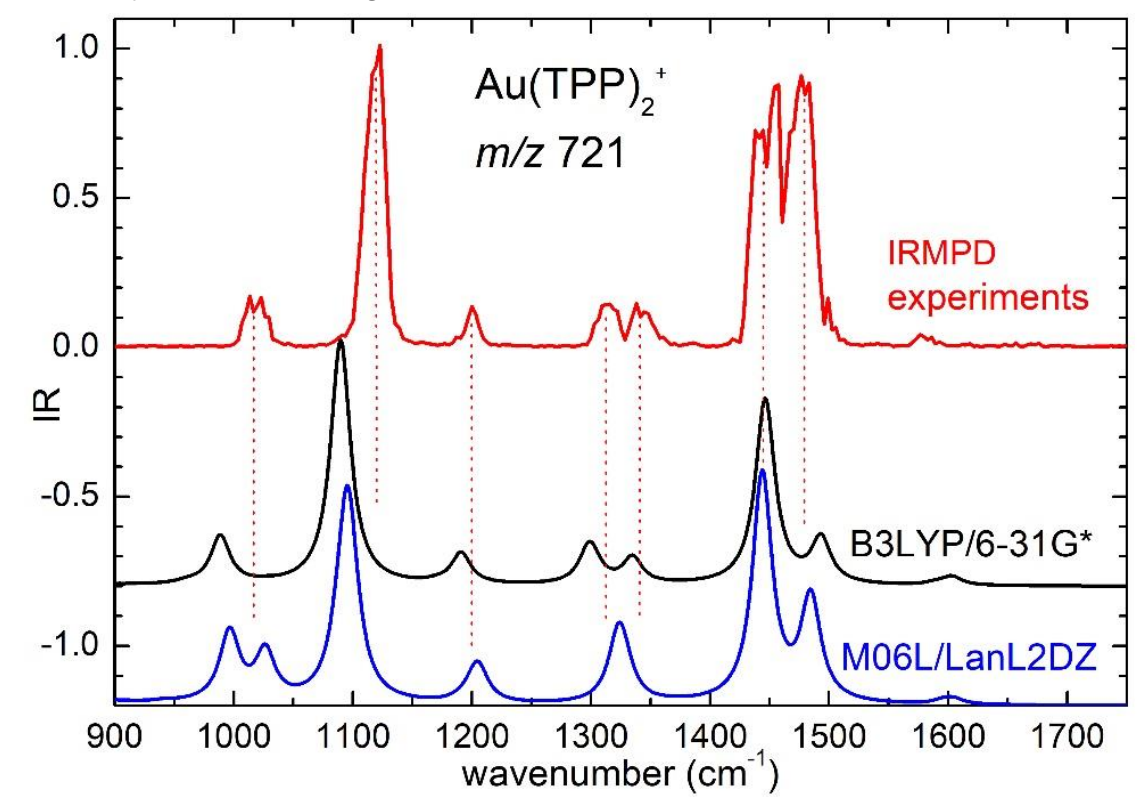

FIG. 4: Comparison between experimental IRMPD spectrum (red) and theoretical spectra, calculated at the M06L/LanL2DZ (blue) and B3LYP/6-31G*levels (black), for $\left[\mathrm{Au}(\mathrm{TPP})_{2}\right]^{+1}$ ions.

For this simple ion whose equilibrium structure is straightforward (see Fig. 3 in the supplementary material), both B3LYP and M06L calculations much better fit with the experimental spectrum, with all experimental features that are well reproduced both in frequency and in intensity. The only two noticeable discrepancies are for the experimental peak at $1480 \mathrm{~cm}^{-1}$ (aromatic C-C stretches), which is calculated too blue-shifted by $5-10 \mathrm{~cm}^{-1}$ and of too low intensity, and for the peak at $1120 \mathrm{~cm}^{-1}$ (PC stretch coupled to aromatic $\mathrm{C}-\mathrm{H}$ in-plane bend) which is too red-shifted by $25-30 \mathrm{~cm}^{-1}$ in both calculations. This last discrepancy is not so surprising since it has been already observed in several previous comparisons involving stretching modes with phosphorus atoms for which the usual scaling factors often lead to too low calculated frequencies [Correia][Scuderi]. In this simple test case there 
are much less IR-active groups, particularly no more amide groups, but the remaining absorption features, which here correspond to internal phenyl and phenyl-phosphorus stretching modes, are well reproduced and this validates the present calculation methodology and the use of a unique scaling factor of 0.975 for both B3LYP and M06L methods. The Au-P distances are equal and a little shorter than in the NC, i.e. $2.39 \AA$ instead of $2.45 \AA$. For the unique gold atom, the NPA charge is now slightly positive, close to +0.2 , while the two phosphorus atoms still hold a partial charge close to +1 and each phenyl group are slightly negative (about -0.2 ), so that the total charge +1 is again mainly brought by the two TPP ligands, more or less equally (+0.4 each).

\section{Gold-glutathione cyclic complexes}

Figure 5 displays a comparison between IRMPD spectra obtained with [(AuSG) $\left.)_{10}-4 \mathrm{H}\right]^{4-}$ anions at $m / z$ 1257 and the FTIR spectrum on the solid neutral sample in $\mathrm{KBr}$ pellets. In contrast with the previous TPP NC, these two spectra are quite different. In the Amide range, the FTIR spectrum displays three main bands: a double one at 1640 and $1600 \mathrm{~cm}^{-1}$, and two others at 1525 and $1400 \mathrm{~cm}^{-1}$. The IRMPD spectrum displays also three wide bands: Amide I at $1725-1765 \mathrm{~cm}^{-1}$, i.e. more than $100 \mathrm{~cm}^{-1}$ above the FTIR doublet; Amide II at 1480-1590 $\mathrm{cm}^{-1}$, which corresponds quite well to the second FTIR band but which is even wider; Amide III band at $1315-1405 \mathrm{~cm}^{-1}$, which is red-shifted by about $40 \mathrm{~cm}^{-1}$ as compared to the third FTIR band and also wider than it. Moreover, the IRMPD spectrum possesses another wide and intense band at $1115-1205 \mathrm{~cm}^{-1}$, in a region where the FTIR spectrum is flat, and below $1000 \mathrm{~cm}^{-1}$ there are only very weak and wide features that do not correspond between the two spectra. We also performed other FTIR experiments, using the Attenuated Total Reflectance (ATR) technique on a thin film of solid sample deposited on a ZnSe window, but with similar spectral features. It is thus likely that the structure of the deprotonated ions studied by IRMPD is quite different from the structure of the solid samples. In particular, the position of the Amide I band is a clear indication that there are much more free amide groups that are not $\mathrm{H}$-bonded in the isolated deprotonated anion as compared to the solid state.

Figure 5 also displays calculated spectra resulting from static harmonic calculations at the M06L/LanL2DZ and B3LYP/6-31G* levels, using the same scaling factor of 0.975 in both cases. This has been performed for two different ring isomers, as displayed in figure 6: the crown structure and 
the catenane one, which were identified in previous studies for similar species [Bertorelle, Liu]. As it can be seen from this comparison, B3LYP calculated spectra (solid lines) reproduce quite well the overall experimental spectrum, while M06L calculations (dotted lines) again fail to reproduce correctly the experimental Amide I band (1665 $\mathrm{cm}^{-1}$ instead of $\left.1745 \mathrm{~cm}^{-1}\right)$ and also miss the experimental band at $1115-1205 \mathrm{~cm}^{-1}$. The differences between the calculated spectra corresponding to the two structures considered here are not so large and sometimes lower than those with experimental data, but a couple of characteristic features tend to favor the crown geometry against the catenane one: (i) the specific experimental band at $1115-1205 \mathrm{~cm}^{-1}$ appears only in the B3LYP calculated spectrum of the crown isomer at the right frequency but with a lower intensity; (ii) the experimental Amide II band $\left(1480-1590 \mathrm{~cm}^{-1}\right)$ is much better reproduced in intensity in the crown B3LYP spectrum. This tentative assignment is however in contradiction with the large free-energy difference of $+230 \mathrm{~kJ} / \mathrm{mol}$ that is calculated between the crown and catenane anion structures and it is also in contradiction with the conclusions of the previous studies on the same species in the $\mathrm{X}$ and UV range [Bertorelle].

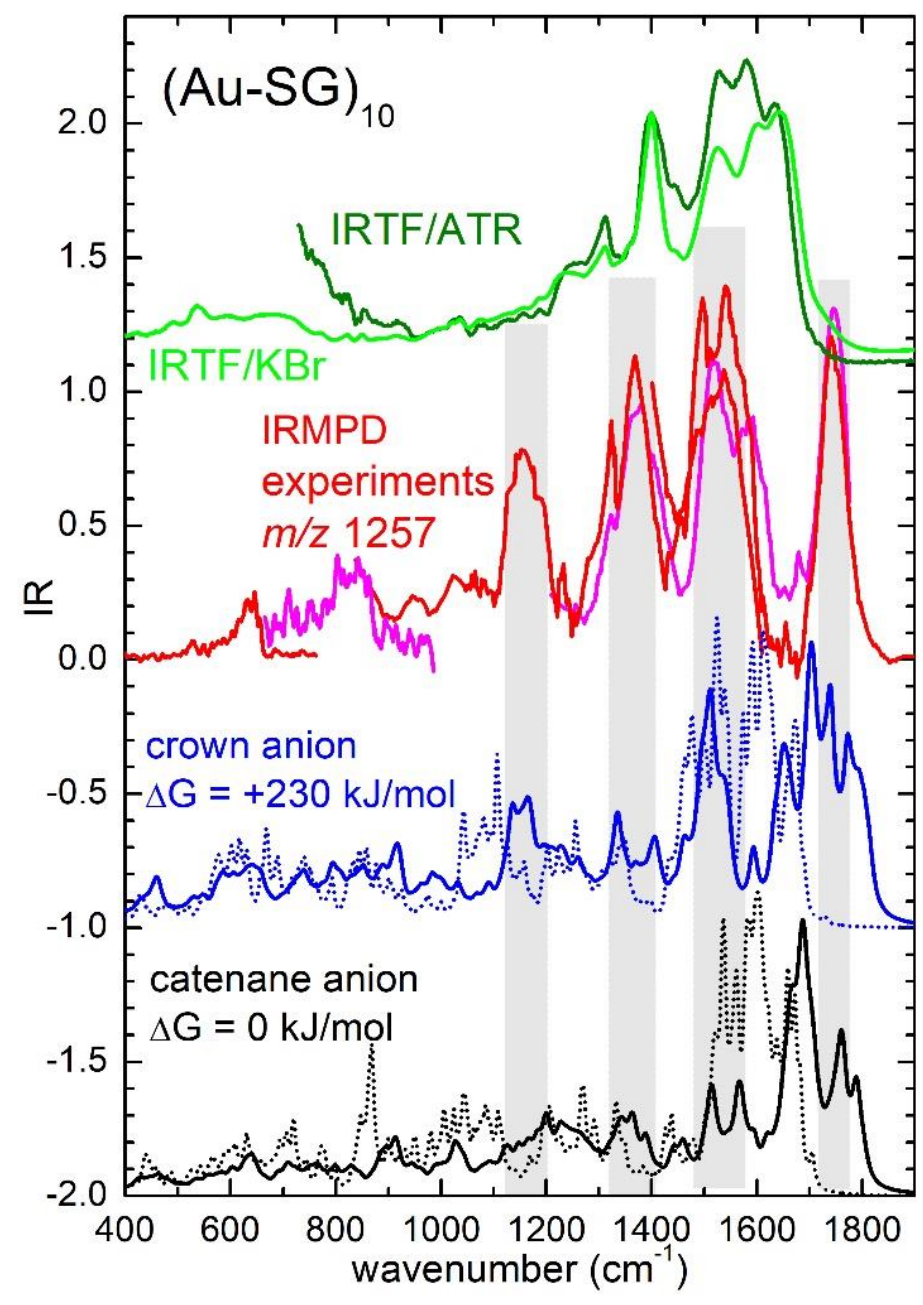

FIG. 5: Experimental IRMPD spectra for $\left[(\text { AuSG }){ }_{10}-4 \mathrm{H}^{+}\right]^{4-}$ (red and magenta curves) at $\mathrm{m} / \mathrm{z} 1257$, compared to FTIR spectrum (green curves) obtained in $\mathrm{KBr}$ pellets and with the ATR technique on the solid neutral samples. These experimental data are also compared to calculated spectra of the crown (blue) and catenane (black) conformations that are performed at the B3LYP/6-31G* (solid) and M06L/LanL2DZ (short dot) levels. As in the previous NC, this last method fails to reproduce the Amide I band at $1745 \mathrm{~cm}^{-1}$. See text for discussion. 
The two calculated equilibrium structures are displayed on figure 6 , as it results from B3LYP/6-31G* calculations and similar equilibrium structures are found from M06L/LanL2DZ calculations, although the crown structure is more flattened in that case. Note that, unlike previous calculations [Bertorelle, Liu], we here took into account all atoms of the glutathione ligands and thus the full interactions between them. However, this total system is too large (almost 400 atoms) to perform a conformational search of the ligand structures and we here present the equilibrium structures that we obtained from one specific geometry optimization for each isomer. In both structures, all the AuS distances are similar but they are slightly shorter and less dispersed in the crown isomer, i.e. in between 2.38 and $2.40 \AA$, as compared to the catenane isomer, where they lie in between 2.37 and 2.47 $\AA$. This is probably due to the fact that there are less constraints on the Au-S chain in the crown isomer that is less compact as compared to the catenane isomer. The same remark holds for the NPA charges, which are much more homogeneous in the crown isomer, i.e. $+0.25 /+0.29$ for Au and $-0.23 /-0.31$ for $\mathrm{S}$ atoms, as compared to the catenane isomer, i.e. $-0.02 /+0.29$ for $\mathrm{Au}$ and $-0.15 /-0.25$ for $S$ atoms. The total charge held by the ten Au-S chain bonds is almost zero $(+0.06)$ for the crown isomer and slightly negative $(-0.3)$ for the catenane isomer, so that the overall -4 charge comes mainly from the four deprotonated glutathione ligands in both cases.
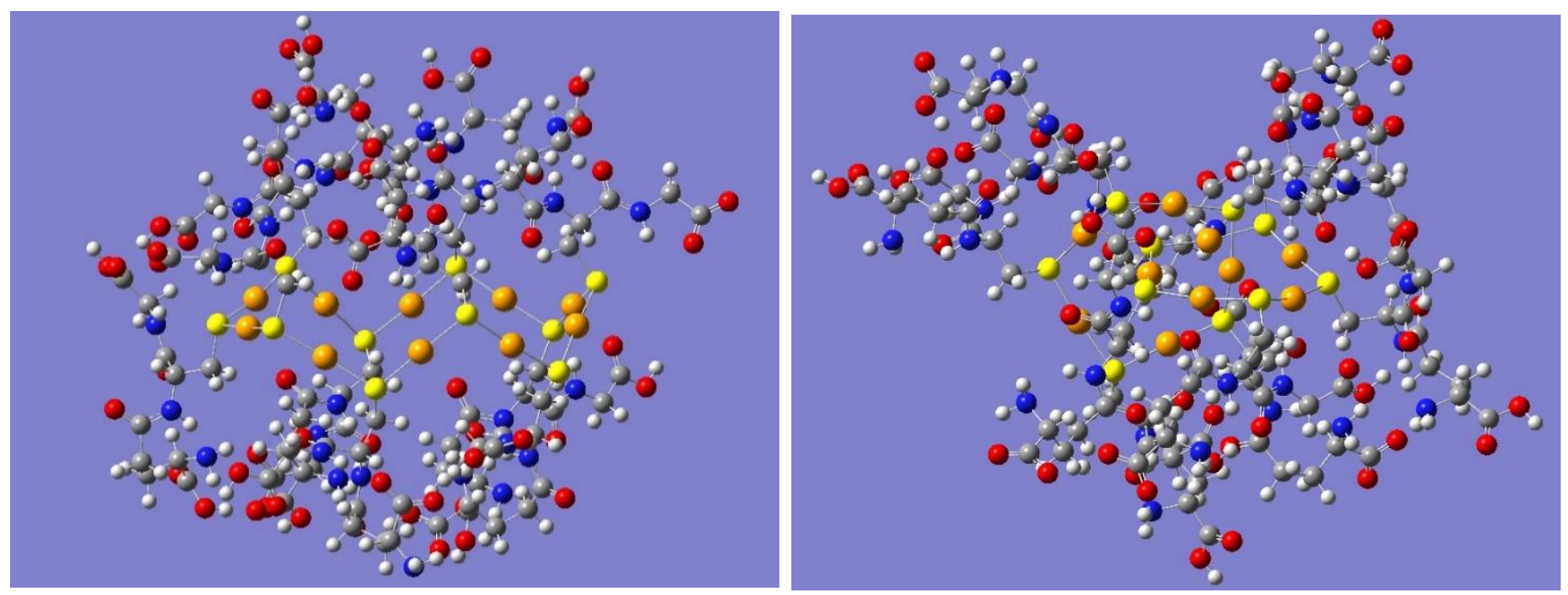

FIG. 6: Theoretical crown (left) and catenane (right) equilibrium structures of [(AuSG) $\left.{ }_{10}-4 \mathrm{H}\right]^{4-}$ isolated ions after full optimization and frequency calculations at the B3LYP/6-31G* level. In both cases, Au-S bonds form a unique (crown) or a double (catenane) cyclic chain on which the ten glutathione ligands are bound via their sulfur atom, with four of them that are deprotonated on their carboxyl side. See text for discussion.

Figure 7 displays experiments and calculations performed on the mono-anion at $m / z 502$ which is made of only one gold atom bound to one deprotonated glutathione ligand, i.e. [AuSG-H $\left.\mathrm{H}^{+}\right]^{-1}$. The experimental spectrum displays wide bands, most probably due to the fact that this ion results mainly from remaining CID processes in our source. Moreover, even for this simple ion, there are many 
possible equilibrium conformations depending on the position of the flexible tripeptide ligand. We thus first performed a conformational search, using the PM6 force field [Stewart], by varying all possible dihedral angles and then grouping conformers by energy and structure families. From a selection of about 20 low-energy families, we then performed full optimizations using DFT, followed by scaled harmonic frequency calculations. Figure 7 displays the structures and the spectra, which have here been convoluted by a larger Lorentzian profile of $20 \mathrm{~cm}^{-1} \mathrm{FWHM}$ in order to take into account the hot character of this ion, for the three lowest conformers calculated at the B3LYP/6$31 \mathrm{G}^{*}$ level. Calculated spectra at the M06L/LanL2DZ level (see Fig. 4 of the supplementary material) more poorly fit with the experimental data, especially again in the Amide region.
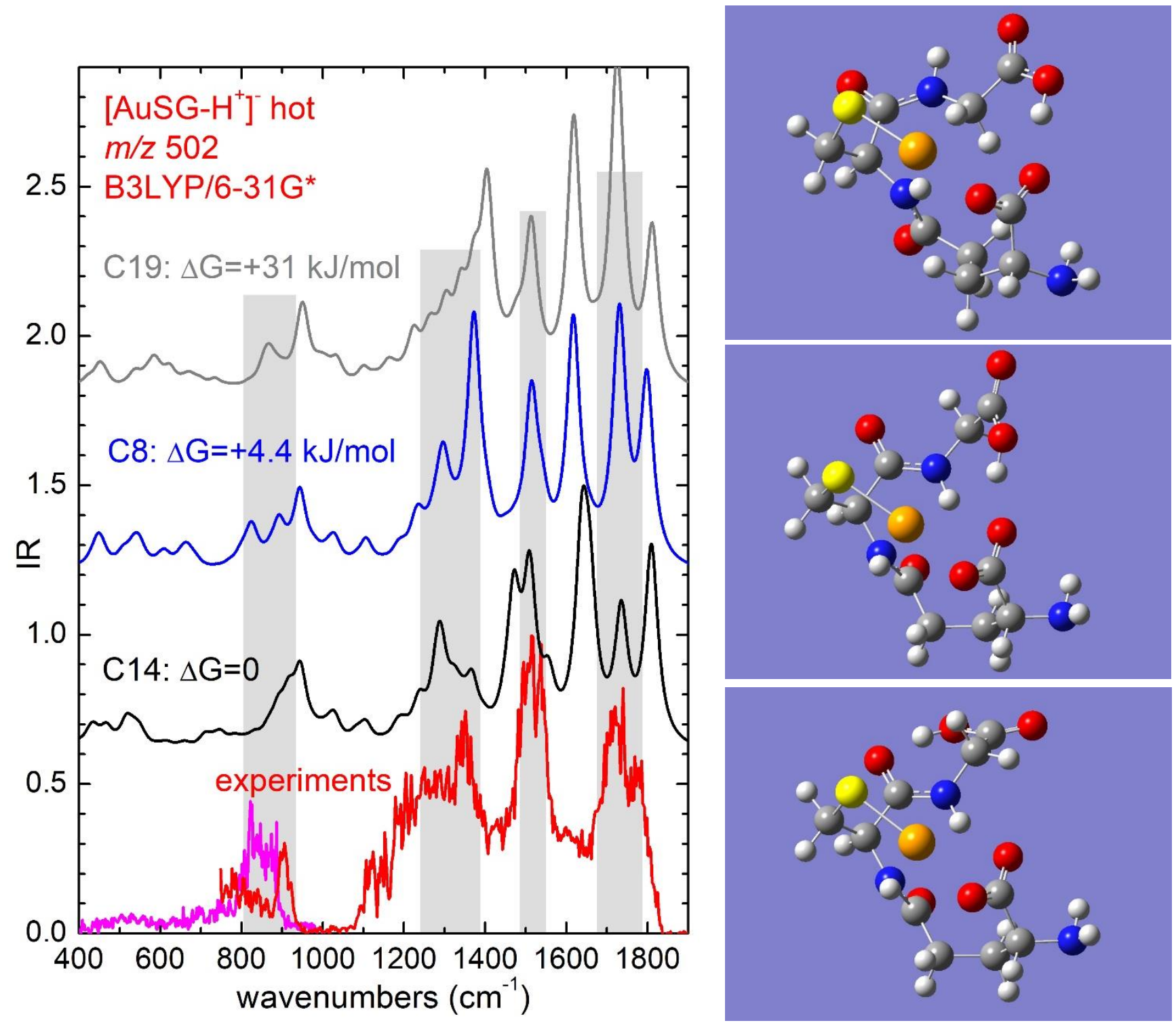

FIG. 7: Comparison between experimental IRMPD spectrum (red and magenta) and theoretical scaled harmonic spectra calculated at the B3LYP/6-31G* level, for the three low-energy conformers of $\left[\text { AuSG- } \mathrm{H}^{+}\right]^{-1}$ ions, which are displayed on the right (C14: bottom and black; C8: middle and blue; $\mathrm{C} 19$ : top and grey). 
B3LYP calculations of the three low-energy conformers reproduce quite well the experimental spectrum but still with some discrepancies: the stretching modes of the carboxyl, the two amide groups and the carboxylate $\mathrm{C}=\mathrm{O}$ are calculated respectively at 1805,1730 and $1620-1650 \mathrm{~cm}^{-1}$ but this last mode does not appear in the experimental spectrum in this frequency range. The following bands, calculated at $1515 \mathrm{~cm}^{-1}$ and in the range $1250-1380 \mathrm{~cm}^{-1}$ and which respectively correspond to the in-plane $\mathrm{N}-\mathrm{H}$ bend and the in-plane $\mathrm{O}-\mathrm{H}$ bend, reproduce quite well the corresponding experimental bands, particularly for the $\mathrm{C} 14$ and C8 isomers. The last experimental very wide band, in between 810 and $930 \mathrm{~cm}^{-1}$, is also better fitted by the calculated C8 isomer spectrum and corresponds to the $\mathrm{OH}$ and $\mathrm{NH} 2$ wagging modes. Since the $\mathrm{C} 8$ and $\mathrm{C} 14$ isomers are calculated at similar free energies $(\Delta \mathrm{G}=4.4 \mathrm{~kJ} / \mathrm{mol})$, it is likely that they both contribute to the experimental spectrum, while the following C19 isomer (and higher-energy ones) may not contribute too much to the present experimental spectrum

The Au-S distance is calculated to be $2.33 \AA$, i.e. again slightly shorter than in the NC, and there is also a strong interaction between the gold atom and one of the two carboxylate oxygen atoms with distances Au-O that are calculated to be only 2.16-2.17 $\AA$ in C8 and C14 isomers and $2.145 \AA$ in C19 isomer. The other carboxylate oxygen is involved either in a $\mathrm{N}-\mathrm{H}$...O hydrogen bond $(1.88 \AA$ in $\mathrm{C} 14$ and $1.99 \AA$ in $\mathrm{C} 8)$ and/or in a O-H...O hydrogen bond (1.755 $\AA$ in C8 and $1.78 \AA$ in C19). For the unique gold atom, the NPA charge is close to +0.2 for both isomers, i.e. close to the charge of gold atoms in the previous crown isomer, while the sulfur atom still holds a partial charge close to 0.3 , slightly more negative than in the $\mathrm{NC}$, and the total charge -1 is again mainly held by the deprotonated carboxylate group of the ligand (-0.65). 


\section{CONCLUSION}

In this work, we have performed IRMPD spectroscopy experiments on covalently bound metal-ligand nanoclusters containing eleven or ten gold atoms and seven to ten ligands. We have shown that the IRMPD spectral signatures of their vibrational modes, in the frequency range in between 500 and $1800 \mathrm{~cm}^{-1}$, are compatible with both the FTIR spectra of their neutral solid samples and with the IRMPD spectra of the isolated building blocks that constitute them. However, IRMPD spectra potentially display more structural information on mass-selected and isolated species, especially about the ligands configurations in the corresponding fingerprint region. In further experiments, it would be interesting to test whether even lower-frequency spectra $\left(200-500 \mathrm{~cm}^{-1}\right)$ could be obtained and would display signatures that would be more characteristic of metal-ligand and metal-metal interactions too.

Concerning IR spectra simulations, these quite large species (total of about 400 atoms) are at the edge of the possibility of high-precision IR spectra simulations with quantum chemistry methods. In this work, we used DFT calculations with two different functionals (B3LYP, M06L), rather small basis sets $\left(6-31 \mathrm{G}^{*}\right.$ and LanL2DZ), scaled harmonic frequency calculations and one process of optimization but numerical frequency calculations already last about three months using ten processors on a single node. B3LYP/6-31G* calculations lead to simulated spectra that fit well with experimental data, although the discrepancies are of the same order as the spectral differences between possible isomers so that the assignment of one specific conformation to the experimental data is only tentative. Concerning M06L/LanL2DZ calculations, we confirmed that this method fails to lead to a correct spectral description of the $\mathrm{C}=\mathrm{O}$ and Amide region, in the range $1400-1800 \mathrm{~cm}^{-1}$. This conclusion is in good agreement with previous compared theoretical studies with similar functionals and species [Katari, Minenkov].

Further experimental and theoretical work would be necessary in order to further test the above provisional conclusions and to progress towards a better description of nanoclusters that are relevant to characterize the physico-chemical properties of true metal-ligand nanoparticles. More precise experimental data, particularly on cooled ions at shorter wavenumbers (below $500 \mathrm{~cm}^{-1}$ ) and with a higher resolution, should be able to provide more insight into the metallic structure and the metalligand interactions. 


\section{SUPPLEMENTARY MATERIAL}

Supplementary material includes full mass spectra of the samples studied, calculated equilibrium geometry of $\mathrm{Au}(\mathrm{TPP})_{2}$ cations and M06L simulated IR spectra of Au-SG anions.

\section{DATA AVAILABILITY}

The data that support the findings of this study are available from the corresponding author upon reasonable request.

\section{ACKNOWLEDGMENTS}

We are grateful the MAGI HPC center at Université Sorbonne Paris Nord and its engineer Nicolas Grenèche.

We also thank the CLIO facility and its technical staff, in particular Estelle Loire, Fabrice Gobert and Nicolas Jestin for their efficient assistance. The French FT-ICR network (CNRS, FR 3624) is also acknowledged for the access to the MS setup.

Finally, we are also grateful to Frédéric Geinguenaud for the use and its assistance in the FTIR experiments and to Franck Bertorelle and Clothilde Comby-Zerbino for providing us the Au-SG samples. 


\section{REFERENCES}

[Antoine] R. Antoine; Atomically precise clusters of gold and silver: Towards a new class of nonlinear optical nanomaterials, Frontier Research Today 2018, 1, 01001. | Page 1 of 11

[Bakker] J.M. Bakker; T. Besson; J. Lemaire; D. Scuderi; P. Maitre; Gas-phase structure of a $\pi$-AllylPalladium complex: efficient infrared spectroscopy in a 7 T Fourier transform mass spectrometer, J. Phys. Chem. A 2007, 111, 13415-13424.

[Becke] A. D. Becke; A new mixing of Hartree-Fock and local density - functional theories, J. Chem. Phys. 1993, 98,1372-1377.

[Bertorelle] F. Bertorelle et al.; Au10(SG)10: A Chiral Gold Catenane Nanocluster with Zero Confined Electrons. Optical Properties and First-Principles Theoretical Analysis, J. Phys. Chem. Lett. 2017, 8, 1979-1985.

[Cirri] A. Cirri, H. Morales Hernandez, C. Kmiotek, C.J. Johnson; Systematically Tuning the Electronic Structure of Gold Nanocluster through Ligand Derivatization, Angew. Chem. Int. Ed. 2019, $58,13818-13822$.

[Cobley] C.M. Cobley; J. Chen; E.C. Cho; L.V. Wang; Y. Xia; Gold nanostructures: a class of multifunctional materials for biomedical applications, Chem. Soc. Rev. 2011, 40, 1, 44-56.

[Correia] C.F. Correia; P.O. Balaj; D. Scuderi; P. Maitre; G. Ohanessian; Vibrational Signatures of Protonated, Phosphorylated Amino Acids in the Gas Phase, J. Am. Chem. Soc. 2008, 130, 3359.

[Cortie] M.B. Cortie; A.M. McDonagh; Synthesis and optical properties of hybrid and alloy plasmonic nanoparticles; Chem. Rev. 2011, 111, 6, 3713-3725.

[Cramer] C.J. Cramer; D.G. Truhlar; Density functional theory for transition metals and transition metal chemistry, Phys. Chem. Chem. Phys. 2009, 11, 10757-10816.

[Ditchfield] R. H. W. J. Ditchfield; W. J. Hehre; J. A. Pople; Self- consistent molecular- orbital methods. IX. An extended Gaussian - type basis for molecular - orbital studies of organic molecules, Journal of Chemical Physics 1971, 54, 724-728.

[Frisch] Gaussian 16, Revision C.01, M.J. Frisch, Gaussian, Inc., Wallingford CT, 2016.

[Gabelica] V. Gabelica; F. Rosu; E. De Pauw; J. Lemaire; J. C. Gillet; J. C. Poully; F. Lecomte; G. Grégoire; J.P. Schermann ; C. Desfrancois ; Infrared Signature of DNA G-Quadruplexes in the Gas Phase, J. Am. Chem. Soc. 2008, 130, 1810-1811.

[Gonzalez] A.I. Gonzalez Florez; E. Mucha; Doo-Sik Ahn; S. Gewinner; W. Schöllkopf; K. Pagel; G. von Helden; Charge-Induced Unzipping of Isolated Proteins to a Defined Secondary Structure; Angew. Chem. Int. Ed. 2016, 55, 3295-3299. 
[Greisch] J-F. Greisch, A. Ballester-Caudet, S. Volker Kruppa, Zhen Lei, Quan-Ming Wang, C. Riehn, F. Remacle; Gas-Phase Photoluminescence and Photodissociation of Silver-Capped Hexagold Clusters, J. Phys. Chem. A 2018, 122, 5799-5810.

[Hay] P.J. Hay; W.R. Wadt; Ab initio effective core potentials for molecular calculations. Potentials for the transition metal atoms Sc to $\mathrm{Hg}$, Journal of Chemical Physics 1985, 82, 270-283.

[Hirata] K. Hirata, K. Yamashita, S. Muramatsu, S. Takano, K. Ohshimo, T. Azuma, R. Nakanishi, T. Nagata, S. Yamazoe, K. Koyasu, T. Tsukuda; Anion photoelectron spectroscopy of free $\left[\mathrm{Au}_{25}\left(\mathrm{SC}_{12} \mathrm{H}_{25}\right)_{18}\right]^{\top}$, Nanoscale 2017, 9, 13409-13412.

[Jasikova] L. Jasikova; J. Roithova; Infrared Multiphoton Dissociation Spectroscopy with FreeElectron Lasers: On the Road from Small Molecules to Biomolecules, Chem. Eur. J., 2018, 24, 33743390.

[Jiang] Xingya Jiang; Bujie Du; Yingyu Huang; Jie Zheng; Ultrasmall noble metal nanoparticles: Breakthroughs and biomedical implications; Nano Today 2018, 21, 106-125.

[Jin] Rongchao Jin; Chenjie Zeng; Meng Zhou; Yuxiang Chen; Atomically Precise Colloidal Metal Nanoclusters and Nanoparticles: Fundamentals and Opportunities, Chem. Rev. 2016, 116, 10346-10413.

[Jung] J.H. Jung; J.H. Lee; S. Shinkai; Functionalized magnetic nanoparticles as chemosensors and adsorbents for toxic metal ions in environmental and biological fields; Chem. Soc. Rev. 2011, 40, 4464-4474.

[Katari] M. Katari; E. Nicol; V. Steinmetz; G. van der Rest; D. Carmichael; G. Frison; Improved Infrared Spectra Prediction by DFT from a New Experimental Database; Chem. Eur. J. 2017, 23, $8414-8423$.

[Koyasu] K. Koyasu; K. Hirata; Tatsuya Tsukuda; Characterization of Chemically Modified Gold/Silver Superatoms in the Gas Phase; in Physical Chemistry of Cold Gas-Phase Functional Molecules and Clusters; T. Ebata and M. Fuji Ed., Springer 2019, 223-253.

[Lee] C. Lee; W. Yang; R. G. Parr; Development of the Colle-Salvetti correlation-energy formula into a functional of the electron density, Physical Review B 1988, 37, 785.

[Lin] C.-A.J. Lin; C.-H. Lee; J.-T. Hsieh; H.-H. Wang; J.K. Li; J.-L. Shen; W.-H. Chan; H.-I. Yeh; W.H. Chang; Synthesis of fluorescent metallic nanoclusters toward biomedical application: recent progress and present challenges, J. Med. Biol. Eng. 2009, 29, 276-283.

[Liu] Yao Liu; Zhimei Tian; Longjiu Cheng; Size evolution and ligand effects on the structures and stability of (AuL)n ( $L=\mathrm{Cl}, \mathrm{SH}, \mathrm{SCH}$, $\mathrm{PH} 2, \mathrm{P}(\mathrm{CH}) 2, n=1-13)$ clusters, RSC Adv. 2016, 6 , 4705-4712.

[Lu] Y. Lu; W. Chen; Sub-nanometer sized metal clusters: from synthetic challenges to the unique property discoveries, Chem. Soc. Rev. 2012, 41, 3594-3623. 
[Maitre] P. Maitre; D. Scuderi; D. Corinti; B. Chiavarino; M.E. Crestoni; S. Fornarini; Applications of infrared multiple photon dissociation (IRMPD) to the detection of posttranslational modifications; Chem. Rev. 2020, 120, 3261-3295.

[McKenzie] L.C. McKenzie, T.O. Zaikova, J.E. Hutchison; Structurally Similar TriphenylphosphineStabilized Undecagolds, Au11(PPh3)7Cl3 and [Au11(PPh3)8Cl2]Cl, Exhibit Distinct Ligand Exchange Pathways with Glutathione, J. Am. Chem. Soc. 2014, 136, 13426-13435.

[Minenkov] Y. Minenkov, A. Singstad, G. Occhipinti, V.R. Jensen; The accuracy of DFT-optimized geometries of functional transition metal compounds: a validation study of catalysts for olefin metathesis and other reactions in the homogeneous phase, Dalton Trans. 2012, 41, 5526-5541.

[]Nieuwjaer] N . Nieuwjaer, A. Beydoun, F. Lecomte, B. Manil, F. Cappelluti, L. Guidoni, D. Scuderi, C. Desfrançois; IRMPD spectroscopy and quantum chemistry calculations on mono- and bi-metallic complexes of acetylacetonate ligands with aluminum, iron and ruthenium ions, J. Chem.Phys. 2020, 153, 234303.

[Prazeres] R. Prazeres; F. Glotin; C. Insa; D.A. Jaroszynski; J.M. Ortega; Two-color operation of a free-electron laser and applications in the mid-infrared, Eur. Phys. J. D 1998, 3, 87-93.

[Scuderi] D. Scuderi; E. Bodo; B. Chiavarino; S. Fornarini; M.E. Crestoni; Amino Acid Oxidation: A Combined Study of Cysteine Oxo Forms by IRMPD Spectroscopy and Simulations, Chem. Eur. J. 2016, 22, 17239.

[Shang] Li Shang; Shaojun Dong; G.U. Nienhaus; Ultra-small fluorescent metal nanoclusters: Synthesis and biological applications, Nano Today 2011, 6, 401-418.

[Stewart] J.J.P. Stewart; Optimization of parameters for semiempirical methods V: Modification of NDDO approximations and application to 70 elements, J. Mol. Model. 2007, 13, 1173.

[Veenstra] A.P. Veenstra, L. Monzel, A. Baksi, J. Czekner, S. Lebedkin, E.K. Schneider, T. Pradeep, A-N. Unterreiner, M.M. Kappes; Ultrafast Intersystem Crossing in Isolated $A g_{29}(B D T) 12^{3-}$ Probed by Time-Resolved Pump-Probe Photoelectron Spectroscopy, J. Phys. Chem. Lett. 2020, 11, 26752681.

[Woodin] R.L. Woodin; D.S. Bomse; J.L. Beauchamp; Multi-photon dissociation of molecules with low-power continuous wave infrared-laser radiation, J. Am. Chem. Soc. 1978, 100, 3248-3250.

[Zhang] B. Zhang; B. Yan; Analytical strategies for characterizing the surface chemistry of nanoparticles, Anal. Bioanal. Chem. 2010, 396, 973-982.

[Zhao] Y. Zhao; D.G. Truhlar; The M06 suite of density functionals for main group thermochemistry, thermochemical kinetics, noncovalent interactions, excited states, and transition elements: two new functionals and systematic testing of four M06-class functionals and 12 other functionals; Theo. Chem. Acc. 2008, 120, 215-241. Y. Zhao; D. G. Truhlar; A new local density functional for main- 
group thermochemistry, transition metal bonding, thermochemical kinetics, and noncovalent interactions, J. Chem. Phys., 2006, 125, 194101. 